\title{
Spectral Tau method for solving general fractional order differential equations with linear functional argument
}

\author{
Kamal R. Raslan, Mohamed A. Abd El salam*, Khalid K. Ali and Emad M. Mohamed
}

${ }^{*}$ Correspondence: mohamed_salam1985@yahoo.com Department of Mathematics, Faculty of Science, Al-azhar University, Cairo, Egypt

\begin{abstract}
In this paper, a numerical technique for solving new generalized fractional order differential equations with linear functional argument is presented. The spectral Tau method is extended to study this problem, where the derivatives are defined in the Caputo fractional sense. The proposed equation with its functional argument represents a general form of delay and advanced differential equations with fractional order derivatives. The obtained results show that the proposed method is very effective and convenient.
\end{abstract}

Keywords: Spectral Tau method, Fractional order differential equations with functional argument, Caputo fractional derivatives

2010 Mathematics Subject Classification (MSC): 26A33, 65L05, 65D15

\section{Introduction}

Spectral methods have been developed through the last years for the numerical solutions of fractional differential equations. Compared to other numerical methods, spectral methods give high accuracy and have a wide range of applications in many mathematical problems and physical phenomena [1]. The Chebyshev first kind $T_{n}(x)$ are the most common basis function used with the spectral methods deal many applications in numerical analysis, and numerous studies show the merits of them in various applications[2-7].

In recent years, several studies have used spectral methods to solve delay differential equations of integer order such as, numerical approximations based on Chebyshev polynomials [8], Bernoulli polynomials [9], hybrid of block-pulse functions and Taylor series [10], and Legendre wavelet [11]. Additionally, the numerical solution of delay differential equations of fractional order have been reported by many researchers [12-23]. Differential equations of advanced argument had fewer contributions in mathematics research, compared to delay differential equations, which had a great development in the last decade $[24,25]$. The general form of argument (mixed type equations) have been reported by several mathematicians, where Grbz et al. used Laguerre collocation method for solving Fredholm integro-differential equations with functional arguments [26]. Yuzbasi has reported a solution of the generalized pantograph type delay differential equations with linear functional arguments[27]. Reutskiy used the backward substitution method for multi-point problems with linear Volterra-Fredholm integro-differential equations of the

(c) The Author(s). 2019 Open Access This article is distributed under the terms of the Creative Commons Attribution 4.0 International License (http://creativecommons.org/licenses/by/4.0/), which permits unrestricted use, distribution, and reproduction in any medium, provided you give appropriate credit to the original author(s) and the source, provide a link to the Creative Commons license, and indicate if changes were made. 
neutral type [28]. All previous works considered a generalization of delay and advanced differential equations (a kind of unification) with integer order derivative.

In this paper, we introduce a generalized form of delay and advanced differential equations with fractional order derivative. Our proposed problem is called general fractional order differential equations (GFDEs) with linear functional argument. Now, we consider the GFDEs with linear functional argument as follows:

$$
f\left(x, y(x), D^{v_{i}} y\left(p_{i} x+\xi_{i}\right), y^{(i)}\left(q_{i} x+\tau_{i}\right)\right)=0,
$$

where $a \leq x \leq b, \quad v_{i}>0$ and $n-1<v_{i}<n, i=0,1,2 \ldots, n, \quad q_{i}, p_{i}, \xi_{i}, \tau_{i} \in \Re$ under the following conditions

$$
y^{(i)}(0)=\mu_{i},
$$

where (1) and the subject conditions (2) are general form of delay and advanced differential equation with fractional order.

As we focus on linear equations, we consider $f(x)$ a linear function. Concerning the existence of solutions for delay and advanced differential equations, we refer the readers to references [24] and [25], so the solution of the proposed formula (1) exists. The general formula (1) is chosen to be multi-term of fractional order derivatives and the terms contain linear functional argument which are taken to be multi-term of fractional order derivatives as well. Chebyshev polynomials of the first kind are used here to approximate the solution of the proposed Eq. 1 . The Chebyshev polynomials are defined on $[-1,1]$, so the argument in (1) also in $[-1,1]$. The operational matrices of fractional derivatives are presented and employed to deal with a generalized form with the spectral Tau method. The presented operational matrices are used with the Tau method as a matrix discretization method. The obtained numerical results are compared with other methods, where they show that the proposed method gives good accuracy.

\section{Definitions of fractional derivatives}

In this section, we present notation, definitions, and recall well-known results about fractional differential equations and the Chebyshev polynomial of the first kind.

\section{The Caputo fractional derivative}

Definition 1 The Caputo fractional derivative operator $D^{v}$ of order $v$ is defined in the following form:

$$
D^{v} f(x)=\frac{1}{\Gamma(m-v)} \int_{0}^{x} \frac{f^{(m)}(t)}{(x-t)^{v-m+1}} d t, \quad v>0,
$$

where $m-1<v \leq m, m \in \mathbb{N}, x>0$.

Properties $11-D^{v}(\lambda f(x)+\mu g(x))=\lambda D^{v} f(x)+\mu D^{v} g(x)$, where $\lambda$ and $\mu$ are constants.

$$
\begin{aligned}
& \text { 2- } D^{v} C=0, \quad \text { where } C \text { is aconstant, } \\
& 3-D^{v} x^{n}=\left\{\begin{array}{ll}
0, & \text { for } n \in \mathbb{N}_{0} \text { and } n<\lceil\nu\rceil, \\
\frac{\Gamma(n+1)}{\Gamma(n+1-v)} x^{n-v}, & \text { for } n \in \mathbb{N}_{0} \text { and } n \geq\lceil\nu\rceil,
\end{array} \text {, where }\lceil\nu\rceil\right. \text { denote to the }
\end{aligned}
$$
smallest integer greater than or equal to $v$, and $\mathbb{N}_{0}=\{0,1,2, \ldots\}$. 


\section{Chebyshev polynomials of the first kind}

The Chebyshev polynomials $T_{n}(x)$ of the first kind are orthogonal polynomials of degree $n$ in $x$ defined on the $[-1,1]$

$$
T_{n}=\cos n \theta
$$

where $x=\cos \theta$ and $\theta \in[0, \pi]$.

The polynomials $T_{n}(x)$ be generated by using the recurrence relations

$$
T_{n+1}(x)=2 x T_{n}(x)-T_{n-1}(x), \quad T_{0}(x)=1, \quad T_{1}(x)=x, \quad n=1,2, \ldots
$$

The Chebyshev polynomials $T_{n}(x)$ can be expressed in terms of the power $x^{n}$ in different forms found in [29], one of them is

$$
T_{n}(x)=\sum_{k=0}^{[n / 2]} c_{k}^{(n)} x^{n-2 k}
$$

where

$$
c_{k}^{(n)}=(-1)^{k} 2^{n-2 k-1} \frac{n}{n-k}\left(\begin{array}{c}
n-k \\
k
\end{array}\right), \quad 2 k \leq n .
$$

From the previous relation, we can define that:

- If $n$ is even, we find

$$
T_{n}(x)=T_{2 l}(x)=\sum_{j=0}^{l}(-1)^{l-j} 2^{2 j-1} \frac{2 l}{l+j}\left(\begin{array}{l}
l+j \\
l-j
\end{array}\right) x^{2 j} .
$$

- If $n$ is odd, we can write

$$
T_{n}(x)=T_{2 l+1}(x)=\sum_{j=0}^{l}(-1)^{l-j} 2^{2 j} \frac{2 l+1}{l+j+1}\left(\begin{array}{c}
l+j+1 \\
l-j
\end{array}\right) x^{2 j+1}
$$

Form above we can write $T(x)$ as a general matrix form as [29]

$$
T(x)=X(x) M^{T},
$$

where $T(x)$ and $X(x)$ are matrices have the form:

$$
T(x)=\left[\begin{array}{llll}
T_{0}(x) & T_{1}(x) & \ldots & T_{N}(x)
\end{array}\right], \quad X(x)=\left[\begin{array}{llll}
x^{0} & x^{1} & \ldots & x^{N}
\end{array}\right]
$$

and $M$ is $(N+1) \times(N+1)$ matrix given by 


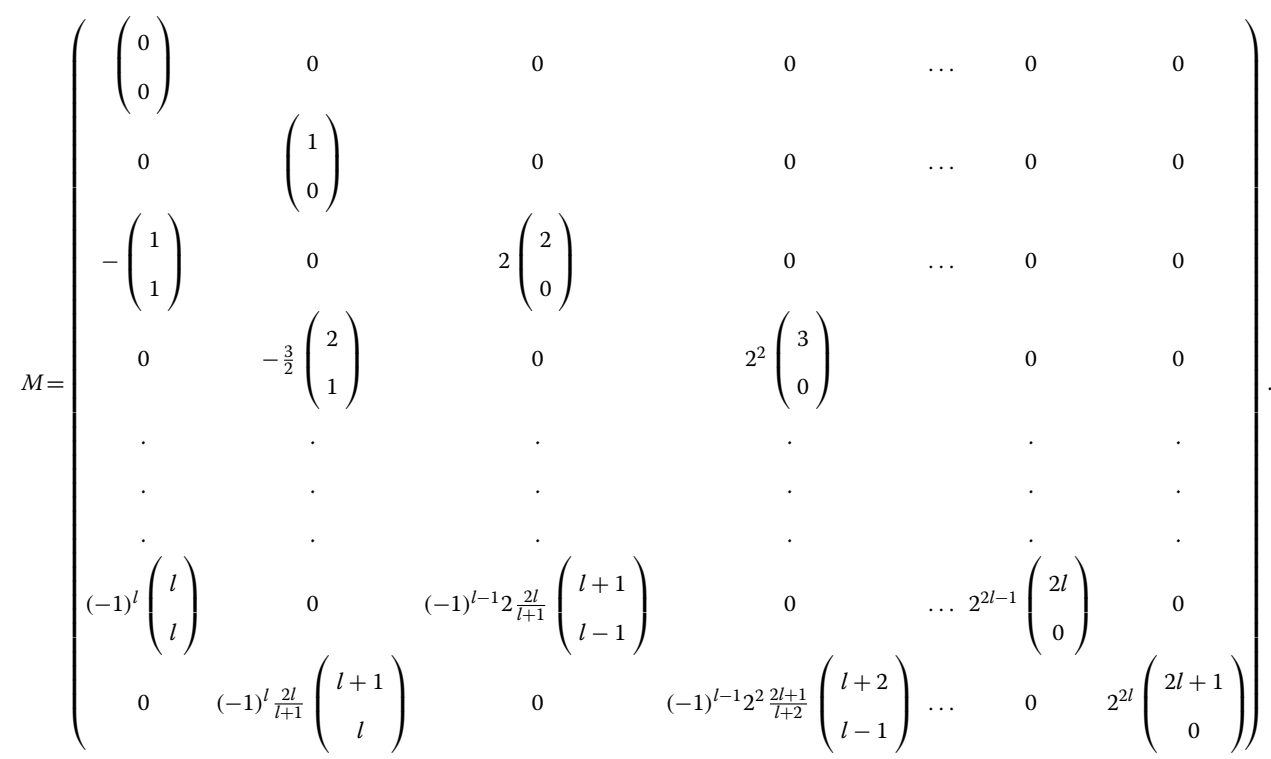

In this case, we are going to use the last row for odd values of $N=2 l+1$, otherwise the previous one will be the last row of matrix $M(N=2 l)$. Now, from (5) we can obtain the $k^{\text {th }}$ derivative of the matrix $T(x)$ as:

$$
T^{(k)}(x)=X^{(k)}(x) M^{T}, \quad k=0,1,2, \ldots
$$

\section{Operational matrices}

In this section, we introduce the operational matrcies for $D^{v_{i}} T\left(q_{i} x+\tau_{i}\right)$ and $T^{(j)}\left(q_{j} x+\tau_{j}\right)$ according to fractional calculus using relations (6) and (5). The $(k)^{\text {th }}$ order derivative of the row vector $T(x)$, can be written in the following relation form [30]:

$$
X^{(k)}(x)=X(x) H^{k}
$$

where $H$ is squar matrix writen as:

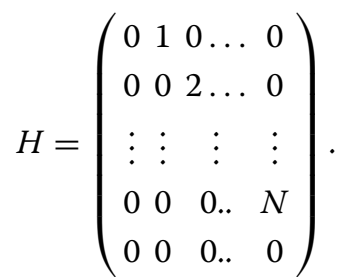

And the row vector $T\left(q_{i} x+\tau_{i}\right)$, represents in terms of the vector $X(x)$ in the following form:

$$
T\left(q_{i} x+\tau_{i}\right)=X\left(q_{i} x+\tau_{i}\right) M^{T}
$$

The $(k)^{\text {th }}$ order derivative of the row vector $T\left(q_{i} x+\tau_{i}\right)$, can be represented as:

$$
\begin{aligned}
T^{(k)}\left(q_{i} x+\tau_{i}\right) & =X^{(k)}\left(q_{i} x+\tau_{i}\right) M^{T} \\
& =X(x) B_{\tau_{i}} H^{k}\left(M E_{q_{i}}\right)^{T}
\end{aligned}
$$

Where the elements of the diagonal matrix $E_{q_{i}}$ can be written as: 


$$
e_{r s}=\left\{\begin{array}{lll}
0 & \text { if } & r \neq s \\
q_{i}^{r} & \text { if } & r=s
\end{array},\right.
$$

where

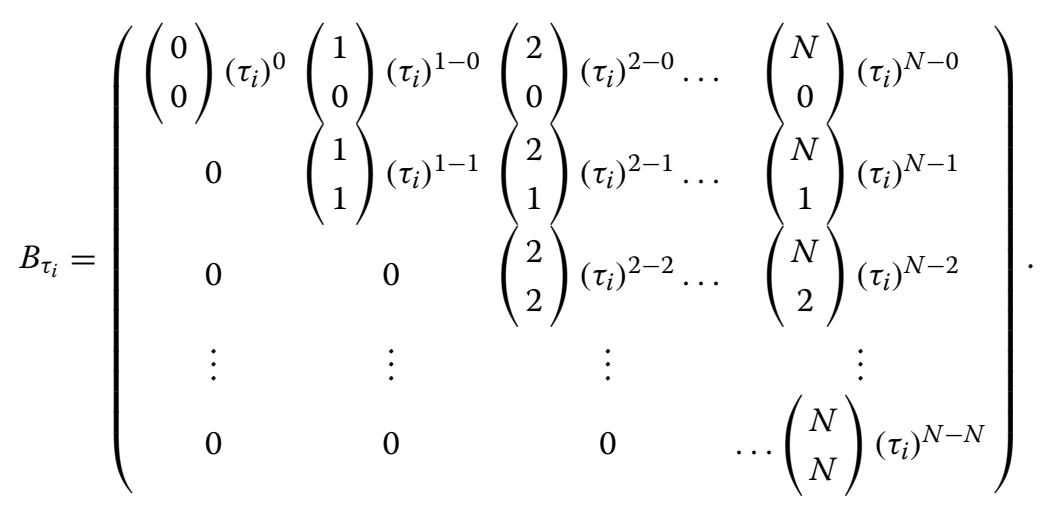

The $v_{i}^{\text {th }}$ order fractional derivative of the vector $T(x)$ can be written as:

$$
D^{\nu_{i}} T(x)=X_{v_{i}}(x) H_{\nu_{i}} M^{T},
$$

where

$$
X_{v_{i}}(x)=\left[0,0, \ldots 0, x^{n-v_{i}}, \ldots . . x^{N-v_{i}}\right], \quad n-1<v_{i}<n, n \in N,
$$

and

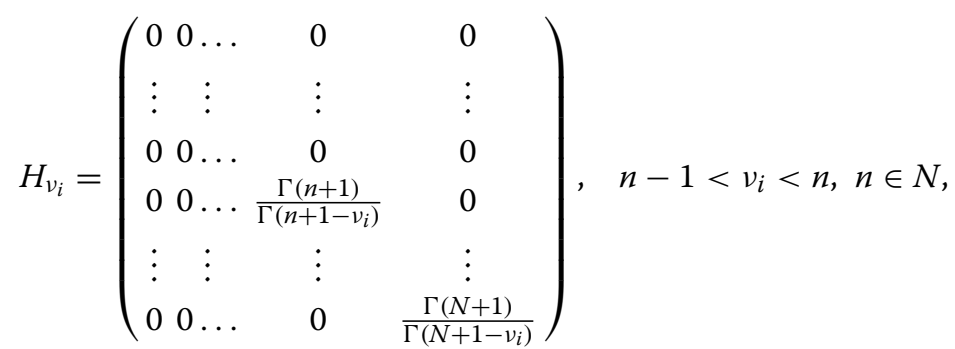

as special case, if $0<v_{i}<1$, then (12) and (13) can written as:

$$
\begin{aligned}
X_{v_{i}}(x)= & {\left[0, x^{1-v_{i}}, x^{2-v_{i}}, \ldots . . x^{N-v_{i}}\right], } \\
H_{v_{i}} & =\left(\begin{array}{cccc}
0 & 0 & 0 \ldots & 0 \\
0 & \frac{\Gamma(2)}{\Gamma\left(2-v_{i}\right)} & 0 \ldots & 0 \\
0 & 0 & \frac{\Gamma(3)}{\Gamma\left(3-v_{i}\right)} \ldots & 0 \\
\vdots & \vdots & \vdots & \vdots \\
0 & 0 & 0 \ldots & \frac{\Gamma(N+1)}{\Gamma\left(N+1-v_{i}\right)}
\end{array}\right) .
\end{aligned}
$$

\section{Application to fractional order differential equation}

In this section, the general form of operational matrices of all terms for (1) and (2) will be obtained. Now, consider the approximate solution according to Chebyshev approximation as:

$$
y(x) \cong y_{N}(x)=\sum_{i=0}^{N} a_{i} T_{i}(x),
$$

where the coefficients $a_{i}$ are given by: 


$$
a_{i}= \begin{cases}\frac{1}{\pi} \int_{-1}^{1} y(x) T_{0}(x) w(x) d x, & \text { if } \quad i=0, \\ \frac{2}{\pi} \int_{-1}^{1} y(x) T_{i}(x) w(x) d x, & \text { if } \quad i=1,2, \ldots, N,\end{cases}
$$

where $w(x)=\frac{1}{\sqrt{1-x^{2}}}$.

Form (16) we get,

$$
y_{N}(x)=T(x) A,
$$

$$
y_{N}^{(i)}\left(q_{i} x+\tau_{i}\right)=T^{(i)}\left(q_{i} x+\tau_{i}\right) A
$$

$$
D^{v_{i}} y_{N}\left(p_{i} x+\xi_{i}\right)=T^{\left(v_{i}\right)}\left(p_{i} x+\xi_{i}\right) A,
$$

where

$$
A=\left[a_{0}, a_{1}, a_{2}, \ldots, a_{N}\right]^{T} .
$$

By using (5) and (18), we get

$$
y_{N}(x)=X(x) M^{T} A,
$$

also, by using (10) and (19), we get

$$
\begin{aligned}
y_{N}^{(i)}\left(q_{i} x+\tau_{i}\right) & =X\left(q_{i} x+\tau_{i}\right) H^{i} M^{T} A \\
& =X(x) H^{i} B_{\tau_{i}}\left(M E_{q_{i}}\right)^{T} A,
\end{aligned}
$$

and by substituting (11) in (20), we get

$$
\begin{aligned}
D^{v_{i}} y_{N}(x)\left(p_{i} x+\xi_{i}\right) & =X\left(p_{i} x+\xi_{i}\right) H_{v_{i}} M^{T} A \\
& =X_{v_{i}}(x) H_{v_{i}} B_{\xi_{i}}\left(M E_{p_{i}}\right)^{T} A
\end{aligned} .
$$

For non-homogeneous term $g(x)$, using Eqs. (5), (16), and (17) can be written in the matrix form as:

$$
\begin{aligned}
G & =X(x) M^{T} A^{\prime} \\
A^{\prime} & =\left(\begin{array}{c}
\frac{1}{\pi} \int_{-1}^{1} g(x) T_{0}(x) w(x) d x \\
\frac{2}{\pi} \int_{-1}^{1} g(x) T_{1}(x) w(x) d x \\
\vdots \\
\frac{2}{\pi} \int_{-1}^{1} g(x) T_{N}(x) w(x) d x
\end{array}\right) .
\end{aligned}
$$

For terms that contain variable coefficients, we may use it in the matrix form as:

$$
Q_{i}(x) \sim\left(\begin{array}{ccccc}
Q_{i}(x) & 0 & 0 & \ldots & 0 \\
0 & Q_{i}(x) & 0 & \ldots & 0 \\
\vdots & \vdots & \vdots & \vdots & \vdots \\
0 & 0 & 0 & \ldots & Q_{i}(x)
\end{array}\right) .
$$

\section{Matrix relation for the conditions}

Finaly, we can obtain the matrix form for the conditions (2) by using (18) on the form:

$$
X(0) H^{i} M^{T} A=\mu_{i}, \quad i=0,1,2 \ldots, m-1 .
$$




\section{Method of solution}

Now, we are ready to construct the fundamental matrix equation corresponding to (1) for this purpose, we substitute (21), (22), and (23), into (1). Thus, we have the fundamental matrix equation as:

$$
f\left(x, X(x) M^{T} A, X(x) H^{i} B_{\tau_{i}}\left(M E_{q_{i}}\right)^{T} A, X_{v_{i}}(x) H_{v_{i}} B_{\xi_{i}}\left(M E_{p_{i}}\right)^{T} A\right)=0,
$$

so, the residual $R(x)$ of Eq. 1, can be written as:

$$
R(x)=f\left(x, X(x) M^{T} A, X(x) H^{i} B_{\tau_{i}}\left(M E_{q_{i}}\right)^{T} A, X_{v_{i}}(x) H_{v_{i}} B_{\xi_{i}}\left(M E_{p_{i}}\right)^{T} A\right) .
$$

As in a typical Tau method, we generate $(N-m+1)$ algebraic equations by applying

$$
\left\langle R(x), T_{i}(x)\right\rangle=\int_{-1}^{1} R(x) T_{i}(x) w(x) d x, \quad i=0,1, \ldots, N-m+1 .
$$

Equations (24) and (27) generate $(m)$ and $(N-m+1)$ set of algebraic equations, respectively. Consequently, the unknown coefficients of the vector $A$ in (16) can be calculated.

\section{Error estimation}

If the exact solution is known, then the error will be estimated from the following:

$$
e_{N}(x)=\left|y(x)-y_{N}(x)\right|
$$

where $y(x)$ is the exact solution and $y_{N}(x)$ is the approximate solution. We can easily check the accuracy of the suggested method by the residual error, since the truncated Chebyshev series (16) is an approximate solution of (1), when the solution $y_{N}(x)$ and its derivatives are substituted in (1), the resulting equation must be satisfied approximately, that is, for $x \in[-1,1], l=0,1,2, \ldots$

$$
e_{N}=\left|f\left(x_{l}, y_{N}\left(x_{l}\right), D^{v_{i}} y_{N}\left(p_{i} x_{l}+\xi_{i}\right), y_{N}^{(i)}\left(q_{i} x_{l}+\tau_{i}\right)\right)\right| \cong 0,
$$

and $e_{N} \leq 10^{\$}$ (\$ positive integer). If $\max 10^{\$}=10^{-L}$ (\$ positive integer) is prescribed, then the truncation limit $N$ is increased until the difference $e_{N}$ at each of the points becomes smaller than the prescribed $10^{L}$. On the other hand, the error can be estimated by the function If $e_{N} \longrightarrow 0$,when $N$ is sufficiently large enough, then the error decreases.

\section{Applications and numerical results}

In this section, we introduce some numerical examples for fractional order differential equation to illustrate the above results. All results are obtained by using Mathematica 7 program.

Example 1 Consider the second-order linear fractional differential equation (mixed type delay-advanced):

$$
y^{\prime \prime}(3 x+2)+y^{\prime \prime}(x)+\left(x^{2}+1\right) D^{\nu} y(x-0.3)+D^{\alpha} y(x)+y(x)=g(x),
$$

the connected conditions are $y(0)=0, y^{\prime}(0)=1, g(x)=20 .+0.445697 x^{0.3}+1.12706 x^{0.4}+$ $x+1.71422 x^{1.3}+1.61009 x^{1.4}+x^{2}+0.445697 x^{2.3}+1.71422 x^{3.3}$ and the exact solution 
when $v=0.7, \alpha=0.6$ is $y(x)=x^{2}+x$. By using the truncated Chebyshev series (16) with the present method, we get algebraic equations using the following residual:

$$
R(x)=\left[X H^{2} B_{2}\left(E_{3} M\right)^{T}+X H^{2} M^{T}+Q_{0} X_{v} H_{v} B_{-0.3} M^{T}+X_{\alpha} H_{\alpha} M^{T}+X M^{T}\right] A-G
$$

where

$$
\begin{aligned}
& X=\left(\begin{array}{c}
1 \\
x \\
x^{2} \\
x^{3} \\
x^{4} \\
x^{5}
\end{array}\right), X_{0.7}=\left(\begin{array}{c}
1 \\
x^{0.3} \\
x^{2.3} \\
x^{3.3} \\
x^{4.3} \\
x^{5.3}
\end{array}\right), X_{0.6}=\left(\begin{array}{c}
1 \\
x^{0.4} \\
x^{2.4} \\
x^{3.4} \\
x^{4.4} \\
x^{5.4}
\end{array}\right), B_{2}=\left(\begin{array}{cccccc}
1 & 2 & 4 & 8 & 16 & 32 \\
0 & 1 & 4 & 12 & 32 & 80 \\
0 & 0 & 1 & 6 & 24 & 80 \\
0 & 0 & 0 & 1 & 8 & 40 \\
0 & 0 & 0 & 0 & 1 & 10 \\
0 & 0 & 0 & 0 & 0 & 1
\end{array}\right) \\
& E_{3}=\left(\begin{array}{cccccc}
1 & 0 & 0 & 0 & 0 & 0 \\
0 & 3 & 0 & 0 & 0 & 0 \\
0 & 0 & 9 & 0 & 0 & 0 \\
0 & 0 & 0 & 27 & 0 & 0 \\
0 & 0 & 0 & 0 & 81 & 0 \\
0 & 0 & 0 & 0 & 0 & 243
\end{array}\right), Q_{0}=\left(\begin{array}{cccccc}
1+x^{2} & 0 & 0 & 0 & 0 & 0 \\
0 & 1+x^{2} & 0 & 0 & 0 & 0 \\
0 & 0 & 1+x^{2} & 0 & 0 & 0 \\
0 & 0 & 0 & 1+x^{2} & 0 & 0 \\
0 & 0 & 0 & 0 & 1+x^{2} & 0 \\
0 & 0 & 0 & 0 & 0 & 1+x^{2}
\end{array}\right), \\
& H_{0.7}=\left(\begin{array}{cccccc}
0 & 0 & 0 & 0 & 0 & 0 \\
0 & 1.11424 & 0 & 0 & 0 & 0 \\
0 & 0 & 1.71422 & 0 & 0 & 0 \\
0 & 0 & 0 & 2.23594 & 0 & 0 \\
0 & 0 & 0 & 0 & 2.71023 & 0 \\
0 & 0 & 0 & 0 & 0 & 3.15143
\end{array}\right), M=\left(\begin{array}{cccccc}
1 & 0 & 0 & 0 & 0 & 0 \\
0 & 1 & 0 & 0 & 0 & 0 \\
-1 & 0 & 2 & 0 & 0 & 0 \\
0 & -3 & 0 & 4 & 0 & 0 \\
1 & 0 & -8 & 0 & 8 & 0 \\
0 & 5 & 0 & -20 & 0 & 16
\end{array}\right), \\
& H_{0.6}=\left(\begin{array}{cccccc}
0 & 0 & 0 & 0 & 0 & 0 \\
0 & 1.12706 & 0 & 0 & 0 & 0 \\
0 & 0 & 1.61009 & 0 & 0 & 0 \\
0 & 0 & 0 & 2.01261 & 0 & 0 \\
0 & 0 & 0 & 0 & 2.36777 & 0 \\
0 & 0 & 0 & 0 & 0 & 2.69065
\end{array}\right), A^{\prime}=\left(\begin{array}{c}
12.0055-0.00901411 i \\
5.92462+0.101376 i \\
1.1573-0.216974 i \\
0.510798+0.172611 i \\
-0.100338-0.0118752 i \\
0.0154373-0.0681158 i \\
0.0503126+0.0151133 i
\end{array}\right) . \\
& H=\left(\begin{array}{llllll}
0 & 1 & 0 & 0 & 0 & 0 \\
0 & 0 & 2 & 0 & 0 & 0 \\
0 & 0 & 0 & 3 & 0 & 0 \\
0 & 0 & 0 & 0 & 4 & 0 \\
0 & 0 & 0 & 0 & 0 & 5 \\
0 & 0 & 0 & 0 & 0 & 0
\end{array}\right), B_{-0.3}=\left(\begin{array}{cccccc}
1 & -0.3 & 0.09 & -0.027 & 0.0081 & -0.00243 \\
0 & 1 & -0.6 & 0.27 & -0.108 & 0.0405 \\
0 & 0 & 1 & -0.9 & 0.54 & -0.27 \\
0 & 0 & 0 & 1 & -1.2 & 0.9 \\
0 & 0 & 0 & 0 & 1 & -1.5 \\
0 & 0 & 0 & 0 & 0 & 1
\end{array}\right)
\end{aligned}
$$

Also, by using the conditions, we can generate two algebraic equations as:

$$
\begin{aligned}
& y(0)=X(0) M^{T} A=0, \\
& y^{\prime}(0)=X(0) B M^{T} A=1,
\end{aligned}
$$

by solving this algebraic equations, we have 
Table 1 Comparison of the values of exact and approximate solutions of the problem (36) for $x$ values for example 2

\begin{tabular}{lllll}
\hline$x$ & $\begin{array}{l}\text { Exact } \\
\text { solution }\end{array}$ & $\begin{array}{l}\text { Present method } \\
(N=5)\end{array}$ & $\begin{array}{l}{[12](N=8),} \\
a=0, b=0\end{array}$ & $\begin{array}{l}{[12](N=8),} \\
a=-0.5, b=0.5\end{array}$ \\
\hline 0 & 0.0000 & 0.0000 & -0.0000 & -0.0000 \\
0.2 & 0.0400 & 0.0400 & 0.0400 & 0.0400 \\
0.4 & 0.1600 & 0.1600 & 0.1600 & 0.1600 \\
0.6 & 0.3600 & 0.3600 & 0.3600 & 0.3600 \\
0.8 & 0.6400 & 0.6400 & 0.6400 & 0.6400 \\
1 & 1.0000 & 1.0000 & 1.0122 & 1.0208 \\
\hline
\end{tabular}

$$
A=\left[\begin{array}{lllllll}
\frac{1}{2} & 1 & \frac{1}{2} & 0 & 0 & 0 & 0
\end{array}\right] .
$$

Then the solution of the problem (30) is

$$
y_{6}(x)=\frac{1}{2} T_{0}(x)+1 T_{1}(x)+\frac{1}{2} T_{2}(x)+0 T_{3}(x)+0 T_{4}(x)+0 T_{4}(x)+0 T_{6}(x)=x^{2}+x,
$$

which is the exact solution of the problem (30).

Example 2 Consider the linear fractional order delay differential equation [31]

$$
D^{\frac{1}{2}} y(x)+y(x)-y(x-1)=2 x+\frac{\Gamma(3)}{\Gamma(1.5)} x^{1.5}-1,
$$

the given condition is $y(0)=0$ and the exact solution is $y(x)=x^{2}$, in [31] the solution obtained by using the shifted Jacobi polynomial scheme, the results are shown by deriving operational matrix for the fractional differentiation and integration. We will employ the present method to (36) at $N=5$.

We get algebraic equations by using the following residual:

$$
R(x)=\left[X_{0.5} H_{0.5} M^{T}+X M^{T}-X B_{-1} M^{T}\right] A-G .
$$

Also, by using the given condition, we can generate algebraic equation as:

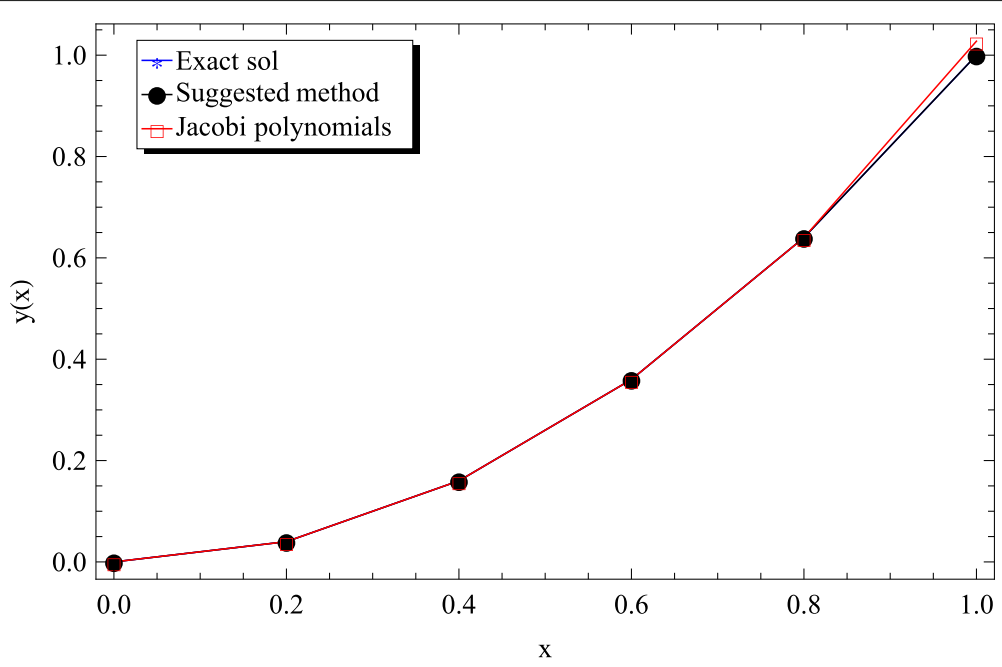

Fig. 1 The behavior of the exact solution and the approximate solution at $N=5$ for example 2 
Table 2 Comparison of the residual errors by using proposed method at different $N$ for $x$ values for example 2

\begin{tabular}{llll}
\hline$x$ & $e_{10}$ & $e_{8}$ & $e_{6}$ \\
\hline 0 & $-8.88 \times 10^{-16}$ & $-3.88 \times 10^{-14}$ & $-5.34 \times 10^{-13}$ \\
0.2 & $-3.93 \times 10^{-16}$ & $-1.80 \times 10^{-14}$ & $-2.48 \times 10^{-13}$ \\
0.4 & $2.15 \times 10^{-16}$ & $7.00 \times 10^{-15}$ & $9.65 \times 10^{-14}$ \\
0.6 & $9.00 \times 10^{-16}$ & $3.49 \times 10^{-14}$ & $4.80 \times 10^{-13}$ \\
0.8 & $1.64 \times 10^{-15}$ & $6.51 \times 10^{-14}$ & $8.95 \times 10^{-14}$ \\
1 & $1.33 \times 10^{-13}$ & $9.72 \times 10^{-14}$ & $2.44 \times 10^{-15}$ \\
\hline
\end{tabular}

$$
y(0)=X(0) M^{T} A=0 .
$$

By solving this algebraic equations, we have

$$
A=\left[\begin{array}{llllll}
\frac{1}{2} & 0 & \frac{1}{2} & 0 & 0 & 0
\end{array}\right] \text {. }
$$

Then, the solution is

$$
y_{5}(x)=\frac{1}{2} T_{0}(x)+\frac{1}{2} T_{2}(x)=x^{2} .
$$

Comparison of the values of the exact and approximate solutions of the problem (36) is given in Table 1 and Fig. 1; in addition, comparison of the residual errors (depended on Eq. 29) by using the proposed method at different $N$ is obtained in Table 2 and Fig. 2.

Example 3 Consider the following fractional delay differential equation [32, 33]:

$$
D^{\alpha} y(x)+y(x)+y(x-0.3)=e^{-x+0.3}, \quad 2<\alpha \leq 3,
$$

with the conditions $y(0)=1, y^{\prime}(0)=-1, \xi_{0}=-0.3$ and the exact solution when $\alpha=3$ is $y(x)=e^{-x}$. By the same way, we get the following residual:

$$
R(x)=\left[X_{\alpha} H_{\alpha} M^{T}+X M^{T}+X B_{-0.3} M^{T}\right] A-G .
$$

Also, by using the subjected conditions we can generate two algebraic equations as:

$$
y(0)=X(0) M^{T} A=1,
$$

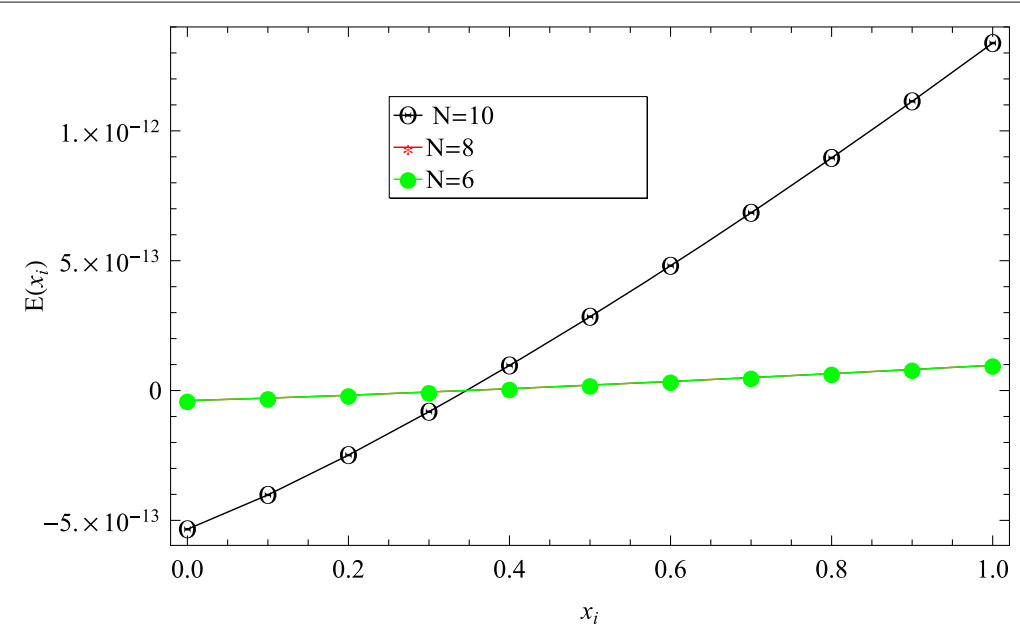

Fig. 2 Residual errors by using the proposed method at different $N$ and $\alpha=0.5$ for example 2 
Table 3 Comparison of the approximate solutions Hermite wavelet method [33], Bernoulli wavelet method [32], and the present method with the exact solution for example 3

\begin{tabular}{|c|c|c|c|c|c|c|}
\hline$x$ & $\begin{array}{l}\text { Exact } \\
\text { solution }\end{array}$ & $\begin{array}{l}\text { Present method } \\
(N=6) \alpha=3\end{array}$ & $\begin{array}{l}{[33](N=7)} \\
\alpha=3\end{array}$ & $\begin{array}{l}{[32](N=7)} \\
\alpha=3\end{array}$ & $\begin{array}{l}\text { Present method } \\
(N=6) \alpha=2.8\end{array}$ & $\begin{array}{l}\text { Present method } \\
(N=6) \alpha=2.6\end{array}$ \\
\hline 0 & 1.0000 & 1.0000 & 1.0000 & 1.0000 & 1.0000 & 1.0000 \\
\hline 0.2 & 0.8187 & 0.8187 & 0.8187 & 0.8187 & 0.8185 & 0.8185 \\
\hline 0.4 & 0.6703 & 0.6703 & 0.6703 & 0.6703 & 0.6685 & 0.6682 \\
\hline 0.6 & 0.5488 & 0.5488 & 0.5488 & 0.5488 & 0.5480 & 0.5488 \\
\hline 0.8 & 0.4493 & 0.4493 & 0.4494 & 0.4493 & 0.4366 & 0.4281 \\
\hline
\end{tabular}

$$
y^{\prime}(0)=X(0) B M^{T} A=-1,
$$

by solving these algebraic equations at $\alpha=3$, we have the solution as:

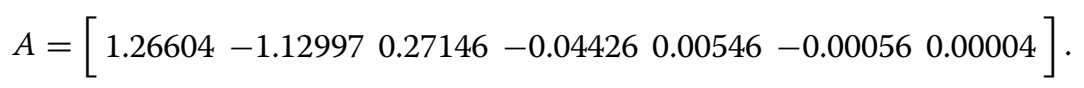

Table 4 displays the residual errors at different $N$, while the approximate solutions obtained for various values of $x$ by using the present method with $N=6$, the Hermite wavelet method [33] for $N=7$ and the Bernoulli wavelet method [32] together with the exact solution are listed in Table 3. Table 3 also contains the numerical results for (41) at $\alpha=2.8$ and 2.6. In addition, Fig. 3 shows the approximate solutions at different $\alpha$ and the exact solution $(\alpha=3)$ and Fig. 4 shows the residual errors at different $N$ (Table 4$)$.

Example 4 Consider the fractional delay differential equation [32, 33]:

$$
D^{\alpha} y(x)-y(x-\tau)+y(x)=\frac{2}{\Gamma(3-\alpha)} x^{2-\alpha}-\frac{1}{\Gamma(2-\alpha)} x^{1-\alpha}+2 \tau x-\tau^{2}-\tau,
$$

with the conditions $y(0)=0, y^{\prime}(0)=0$ and the exact solution is $y(x)=x^{2}-x$ when $\alpha=1$, $\tau=0.001$, by using (16) at $N=7$, then the fundamental matrix equation of the problem is defined by

$$
\left[Q_{0}(x) X_{\alpha} H_{\alpha} M^{T}+Q_{0}^{*}(x) X M^{T}-P_{0}^{*}(x) X H_{-\tau} M^{T}\right] A-G .
$$

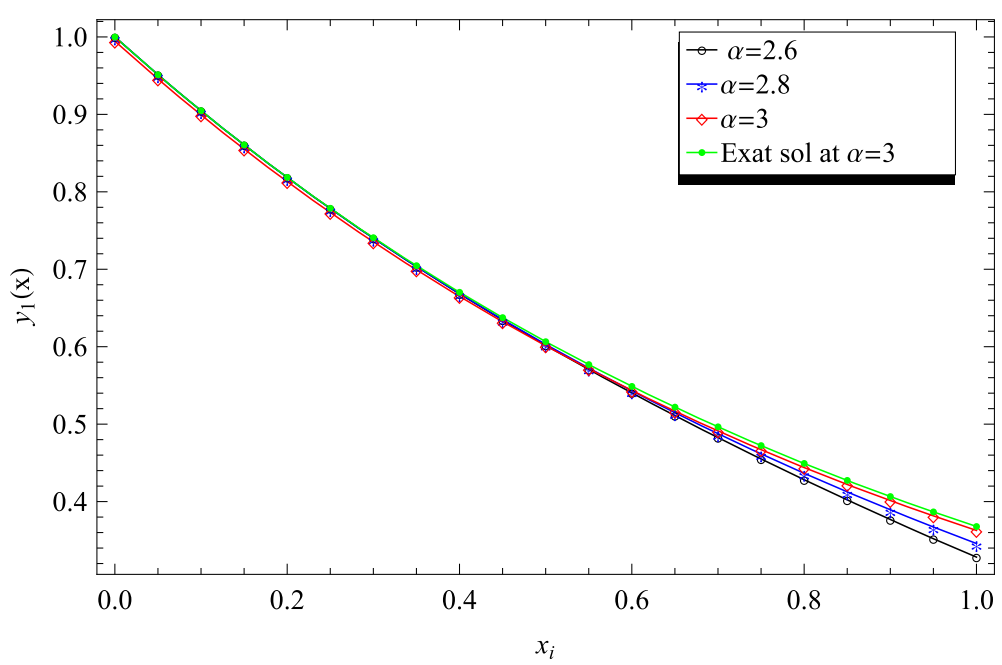

Fig. 3 Solutions by the proposed method at different $\alpha$ and exact solution at $\alpha=3 N=6$ for example 3 


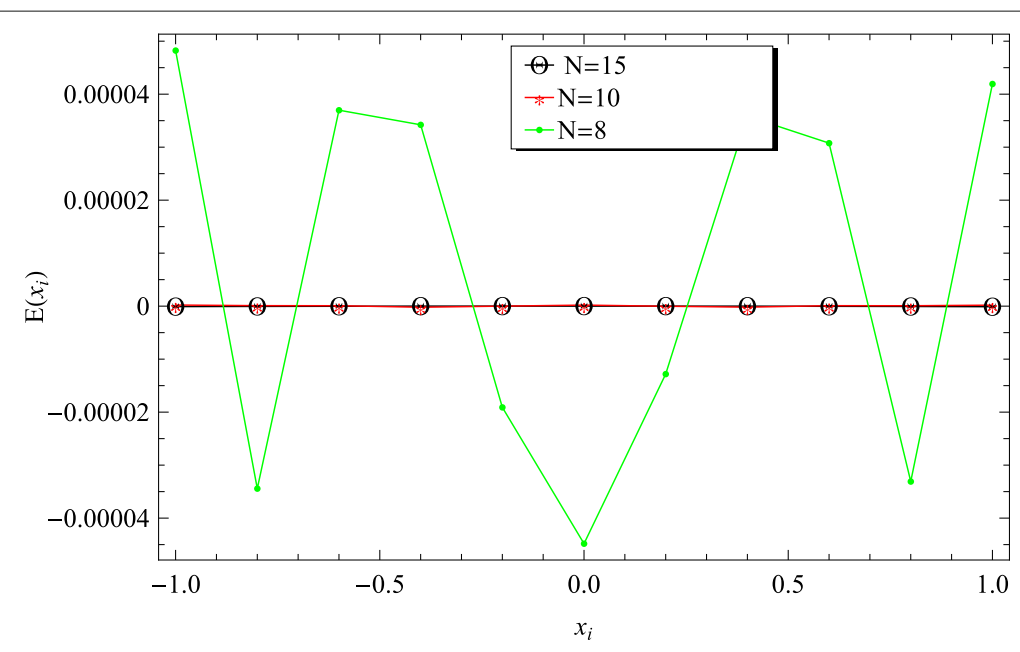

Fig. 4 Residual errors by using the proposed method at different $N$ and $\alpha=3$, for example 3

The numerical results are presented in Table 5, and the absolute errors also listed and compared with Hermite wavelet method [33].

Figure 5 displays the approximate solutions obtained for values of $\alpha=1,0.8,0.7,0.6$, and the exact solution with $N=7$ and $\tau=0.001$. From these results, it is seen that the approximate solutions converge to the exact solution.

Example 5 Consider the fractional order delay differential equation [34]:

$$
D^{\frac{3}{10}} y(x)-y(x-1)+y(x)=1-3 x+3 x^{2}+\frac{2000 x^{2.7}}{1071 \Gamma(0.7)}
$$

The subjected condition $y(0)=0$ and the exact solution is $y(x)=x^{3}$. By using the truncated Chebyshev series (16), then the fundamental matrix equation of the problem is defined by

$$
R(x)=\left[X_{\nu} H_{v} M^{T}+X B_{-1} M^{T}+X M^{T}\right] A-G .
$$

After the augmented matrices of the system and condition are computed, we obtain the coefficient matrix on the form:

Table 4 Comparison of the residual errors by using proposed method at different $N$ and $\alpha=3$ for $x$ values for example 3

\begin{tabular}{llll}
\hline$x$ & $e_{8}$ & $e_{10}$ & $e_{15}$ \\
\hline 0 & $-4.48 \times 10^{-5}$ & $1.98 \times 10^{-5}$ & $1.39 \times 10^{-8}$ \\
0.2 & $-1.28 \times 10^{-5}$ & $-1.82 \times 10^{-8}$ & $7.90 \times 10^{-9}$ \\
0.4 & $3.58 \times 10^{-5}$ & $-1.90 \times 10^{-7}$ & $-6.91 \times 10^{-9}$ \\
0.6 & $7.30 \times 10^{-5}$ & $8.85 \times 10^{-8}$ & $-3.04 \times 10^{-8}$ \\
0.8 & $-3.30 \times 10^{-6}$ & $7.47 \times 10^{-8}$ & $-6.26 \times 10^{-8}$ \\
1 & $4.49 \times 10^{-5}$ & $1.88 \times 10^{-7}$ & $-1.05 \times 10^{-7}$ \\
\hline
\end{tabular}


Table 5 Comparison of the values of exact, approximate solutions for different values of $x$ and the absolute errors at $\tau=0.001$ for example 4

\begin{tabular}{lllll}
\hline$x$ & $\begin{array}{l}\text { Exact } \\
\text { solution }\end{array}$ & $\begin{array}{l}\text { Suggested method } \\
(N=7)\end{array}$ & $\begin{array}{l}\text { absolute error } \\
(N=7)\end{array}$ & $\begin{array}{l}\text { absolute error } \\
{[33]}\end{array}$ \\
\hline 0 & 0.00000 & 0.00000 & 0 & 0 \\
0.2 & -0.16000 & -0.16000 & $5.82 \times 10^{-16}$ & $1.94 \times 10^{-16}$ \\
0.4 & -0.24000 & -0.24000 & $5.55 \times 10^{-16}$ & $3.33 \times 10^{-16}$ \\
0.6 & -0.24000 & -0.24000 & $4.44 \times 10^{-16}$ & $8.60 \times 10^{-14}$ \\
0.8 & -0.16000 & -0.16000 & $3.33 \times 10^{-16}$ & $8.57 \times 10^{-14}$ \\
1 & 0.00000 & 0.00000 & 0 & 0 \\
\hline
\end{tabular}

$$
A=\left[\begin{array}{lllllll}
0 & \frac{3}{4} & 0 & \frac{1}{4} & 0 & 0 & 0
\end{array}\right] .
$$

Then, the solution is of Eq. 48

$$
y_{6}(x)=\frac{3}{4} T_{1}(x)+\frac{1}{4} T_{3}(x)=x^{3},
$$

which is the exact solution of the problem (48).

Example 6 Consider the linear fractional order delay differential equation [35]:

$$
D^{v} y(x)-\frac{1}{2} y^{\prime}(x-\pi)+\frac{1}{2} y(x)=0,
$$

with the initial condition $y(0)=0, y^{\prime}(0)=1$ and the exact solution when $v=2$ is $y(x)=\sin (x)$, which is second-order delay differential equation with oscillatory in nature. By using (16) with $N=6$, we get algebraic equations by using the following residual:

$$
R(x)=\left[X_{\nu} H_{\nu} M^{T}+Q_{1} X H B_{-\pi} M^{T}+Q_{0} X M^{T}\right] A .
$$

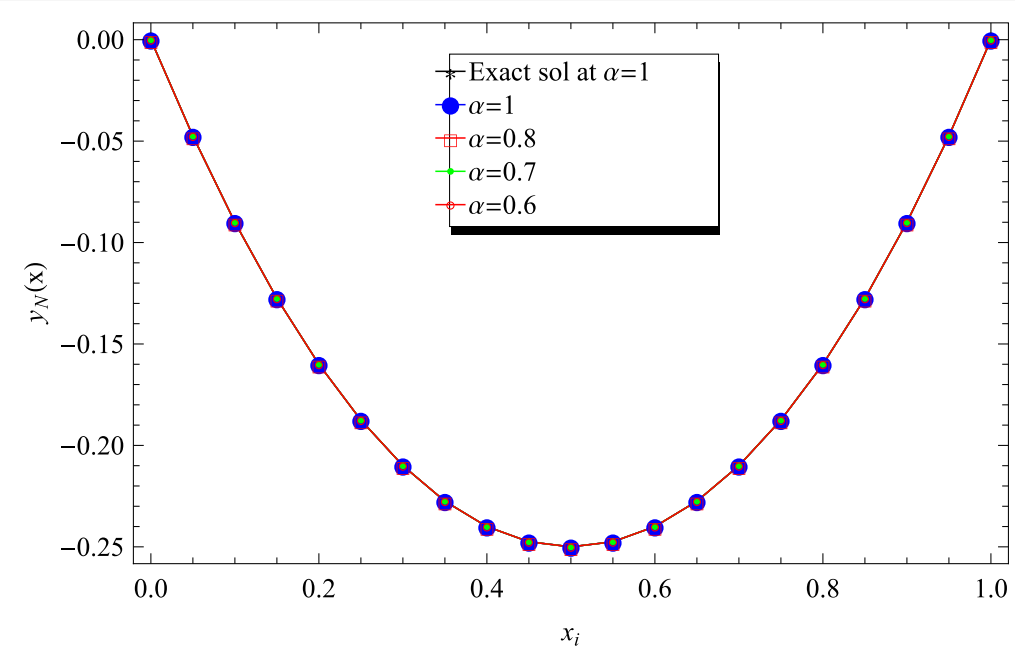

Fig. 5 The comparison of $y_{N}(x)$ for $N=7, \tau=0.001$, with $\alpha=1,0.8,0.7,0.6$ and the exact solution for example 4 
Table 6 Comparison of the values of exact and approximate solutions for $x$ values for example 6

\begin{tabular}{lll}
\hline$x$ & $\begin{array}{l}\text { Exact } \\
\text { solution }\end{array}$ & $\begin{array}{l}\text { present method } \\
(N=6) v=2\end{array}$ \\
\hline 0 & 0.0000 & 0.0000 \\
0.2 & 0.1975 & 0.1985 \\
0.4 & 0.3894 & 0.3892 \\
0.6 & 0.5646 & 0.5645 \\
0.8 & 0.7173 & 0.7173 \\
1 & 0.8414 & 0.8414 \\
\hline
\end{tabular}

Also, by using the initial condition, we can generate two algebraic equations as:

$$
\begin{aligned}
& y(0)=X(0) M^{T} A=0, \\
& y^{\prime}(0)=X(0) B M^{T} A=1,
\end{aligned}
$$

by solving this algebraic system at $v=2$, we have the solution as:

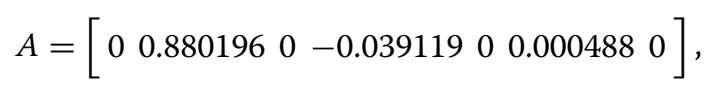

then, the solution of Eq. (48) is:

$$
y_{6}(x)=0.880196 T_{1}(x)-0.039119 T_{3}(x)+0.000488 T_{5}(x) .
$$

Table 6 compares the values of exact and approximate solutions, $x \in[0,1]$, while Table 7 lists the residual errors by using the proposed method at different $N$ and $v=2$.

\section{Conclusion}

In this work, the general form of fractional order differential equations with linear functional argument is presented. The spectral Tau method is used for solving the proposed equation. All terms in the proposed equation reduced by operational matrices based on Chebyshev polynomials to matrix form. The accuracy of this method is obtained by many numerical examples. Finally, we used the Mathematica 7 to calculate our numerical results.

Table 7 Comparison of the residual errors by using the proposed method at different Nand $v=2$ for example 6

\begin{tabular}{llll}
\hline$x$ & $e_{10}$ & $e_{15}$ & $e_{18}$ \\
\hline 0 & $2.35 \times 10^{-3}$ & $-7.17 \times 10^{-4}$ & $-1.95 \times 10^{-4}$ \\
0.2 & $1.88 \times 10^{-4}$ & $-2.52 \times 10^{-4}$ & $-1.03 \times 10^{-4}$ \\
0.4 & $4.72 \times 10^{-4}$ & $-8.16 \times 10^{-5}$ & $-5.25 \times 10^{-5}$ \\
0.6 & $1.89 \times 10^{-4}$ & $-2.38 \times 10^{-5}$ & $-2.50 \times 10^{-5}$ \\
0.8 & $6.93 \times 10^{-5}$ & $-6.2 \times 10^{-6}$ & $-1.10 \times 10^{-5}$ \\
1 & $2.25 \times 10^{-5}$ & $-1.38 \times 10^{-6}$ & $-4.57 \times 10^{-6}$ \\
\hline
\end{tabular}




\section{Abbreviations}

GFDEs: General fractional order differential equations

\section{Acknowledgments}

The authors sincerely thank the editors, reviewers, and everyone who provide advice, support, help, and useful comments. The product of this research paper would not be possible without all of them.

\section{Authors' contributions}

KRR modified the manuscript and arranged it in its final form to meet the suggestions and edits of the referees. MAAES suggested the point for this paper and performed the examples on the computer pacge. KKA revised the mathematical research point and helped in coding using Mathematica program. EMM revised the complete manuscript and the obtained results and was a major contributor in writing the manuscript. All authors contributed to the manuscript writing and read and approved the final manuscript.

\section{Funding}

Not applicable

\section{Availability of data and materials}

The datasets generated and/or analysed during the current study are available from the corresponding author on reasonable request.

\section{Competing interests}

The authors declare that they have no competing interests.

Received: 31 March 2019 Accepted: 26 August 2019

Published online: 11 September 2019

\section{References}

1. Shen, J., Tang, T., Wang, L. L.: Spectral methods: algorithms, analysis and applications, Volume 41 of Series in Computational Mathematics. Springer-Verlag, Berlin, Heidelberg (2011)

2. Maleknejad, K., Nouri, K., Torkzadeh, L.: Operational matrix of fractional integration based on the shifted second kind Chebyshev polynomials for solving fractional differential equations. Mediterr. J. Math. 13(3), 1377-1390 (2016)

3. Bhrawy, A. H., Tharwat, M. M., Yildirim, A.: A new formula for fractional integrals of Chebyshev polynomials: application for solving multi-term fractional differential equations. Appl. Math. Model. 37(6), 4245-4252 (2013)

4. Sweilam, N. H., Khader, M. M., Adel, M.: Chebyshev pseudo-spectral method for solving fractional advection-dispersion equation. Appl. Math. 5(19), 3240 (2014)

5. Khader, M. M., Sweilam, N. H., Mahdy, A. M. S.: The Chebyshev collection method for solving fractional order Klein Gordon equation. Wseas Trans. Math. 13, 2224-2880 (2014)

6. Korkiatsakul, T., Koonprasert, S., Neamprem, K.: A new operational matrix method for solving nonlinear caputo fractional derivative integro-differential static beam problems via Chebyshev polynomials. In: Proceedings of the International MultiConference of Engineers and Computer Scientists, vol. 1, pp. 14-16, (2018)

7. Ramadan, M. A., Abd El Salam, M. A.: Spectral collocation method for solving continuous population models for single and interacting species by means of exponential Chebyshev approximation. Int. J. Biomath. 11(08), 1850109 (2018)

8. Sedaghat, S., Ordokhani, Y., Dehghan, Mehdi: Numerical solution of the delay differential equations of pantograph type via Chebyshev polynomials. Commun. Nonlinear Sci. Numer. Simul. 17(12), 4815-4830 (2012)

9. Tohidi, E., Bhrawy, A. H., Erfani, K.: A collocation method based on Bernoulli operational matrix for numerical solution of generalized pantograph equation. Applied Mathematical Modelling. 37(6), 4283-4294 (2013)

10. Marzban, H. R., Razzaghi, M.: Solution of multi-delay systems using hybrid of block-pulse functions and Taylor series. J. Sound Vib. 292(3-5), 954-963 (2006)

11. Hafshejani, M. S., Karimi Vanani, S., Sedighi Hafshejani, J.: Numerical solution of delay differential equations using Legendre wavelet method. World Appl. Sci. J. 13, 27-33 (2011)

12. Tang, X., Shi, Y., Xu, H.: Well conditioned pseudospectral schemes with tunable basis for fractional delay differential equations. J. Sci. Comput. 74(2), 920-936 (2018)

13. Khader, M. M., Hendy, A. S.: The approximate and exact solutions of the fractional-order delay differential equations using Legendre seudospectral method. Int. J. Pure Appl. Math. 74(3), 287-297 (2012)

14. Wang, Z:: A numerical method for delayed fractional-order differential equations. J. Appl. Math. 2013, 1-7 (2013)

15. Moghaddam, B. P., Mostaghim, Z. S.: A numerical method based on finite difference for solving fractional delay differential equations. J. Taibah Univ. Sci. 7(3), 120-127 (2013)

16. Li, Y., Zhao, W.: Haar wavelet operational matrix of fractional order integration and its applications in solving the fractional order differential equations. Appl. Math. Comput. 216(8), 2276-2285 (2010)

17. Iqbal, M. A., Ali, A., Mohyud-Din, S. T.: Chebyshev wavelets method for fractional delay differential equations. Int. J. Mod. Appl. Phys. 4(1), 49-61 (2013)

18. Khader, M. M., Hendy, A. S.: The approximate and exact solutions of the fractional-order delay differential equations using Legendre seudospectral method. Int. J. Pure Appl. Math. 74(3), 287-297 (2012)

19. Keshavarz, E., Ordokhani, Y., Razzaghi, M.: Bernoulli wavelet operational matrix of fractional order integration and its applications in solving the fractional order differential equations. Appl. Math. Model. 38(24), 6038-6051 (2014)

20. Dehghan, M., Manafian, J., Saadatmandi, A.: Solving nonlinear fractional partial differential equations using the homotopy analysis method. Numer. Methods Partial Diff. Equat. Int. J. 26(2), 448-479 (2010)

21. Saadatmandi, A., Dehghan, M.: A Tau approach for solution of the space fractional diffusion equation. Comput. Math. Appl. 62(3), 1135-1142 (2011) 
22. Saadatmandi, A., Dehghan, M., Azizi, M. R.: The SincLegendre collocation method for a class of fractional convectiondiffusion equations with variable coefficients. Commun. Nonlinear Sci. Numer. Simul. 17(11), 4125-4136 (2012)

23. Dehghan, M., Manafian, J., Saadatmandi, A.: The solution of the linear fractional partial differential equations using the homotopy analysis method. Z. Naturforsh. A. 65(11), 935 (2010)

24. lakovleva, V., Vanegas, C. J.: On the solution of differential equations with delayed and advanced arguments. Electron. J. Differ. Equat. Conf. 13, 57-36 (2005)

25. Rus, A. I., Dârzu-llea, V. A.: First order functional-differential equations with both advanced and retarded arguments. Fixed Point Theory. 5(1), 103-115 (2004)

26. Gürbüz, B., Sezer, M., Güler, C.: Laguerre collocation method for solving Fredholm integro-differential equations with functional arguments, Vol. 2014 (2014)

27. Yüzbai, U., Ismailov, N.: A Taylor operation method for solutions of generalized pantograph type delay differential equations. Turk. J. Math. 42(2), 395-406 (2018)

28. Reutskiy, S. Y.: The backward substitution method for multipoint problems with linear VolterraFredholm integro-differential equations of the neutral type. J. Comput. Appl. Math. 296, 724-738 (2016)

29. Ramadan, M. A., Raslan, K. R., El Danaf, T. S., Abd El Salam, M. A.: An exponential Chebyshev second kind approximation for solving high-order ordinary differential equations in unbounded domains, with application to Dawson's integral. J. Egypt. Math. Soc. 25(2), 197-205 (2017)

30. Gülsu, M., Öztürk, Y., Sezer, M.: A new collocation method for solution of mixed linear integro-differential-difference equations. Appl. Math. Comput. 216(7), 2183-2198 (2010)

31. Xu, M. Q., Lin, Y. Z.: Simplified reproducing kernel method for fractional differential equations with delay. Appl. Math. Lett. 52, 156-161 (2016)

32. Rahimkhani, P., Ordokhani, Y., Babolian, E.: A new operational matrix based on Bernoulli wavelets for solving fractional delay differential equations. Numer. Algoritm. 74(1), 223-245 (2017)

33. Saeed, U.: Hermite wavelet method for fractional delay differential equations. J. Differ. Equ. 2014, 1-8 (2014)

34. Morgado, M. L., Ford, N. J., Lima, P. M.: Analysis and numerical methods for fractional differential equations with delay. J. Comput. Appl. Math. 252, 159-168 (2013)

35. Ahmad, Z. S., Ismail, F., Senu, N.: Solving oscillatory delay differential equations using block hybrid methods. J. Math 2018, 1-7 (2018)

\section{Publisher's Note}

Springer Nature remains neutral with regard to jurisdictional claims in published maps and institutional affiliations.

\section{Submit your manuscript to a SpringerOpen ${ }^{\odot}$} journal and benefit from:

- Convenient online submission

Rigorous peer review

- Open access: articles freely available online

- High visibility within the field

- Retaining the copyright to your article

Submit your next manuscript at $\downarrow$ springeropen.com 